\title{
О ПОНИЖЕНИИ РАЗМЕРНОСТИ ГАМИЛЬТОНОВЫХ СИСТЕМ С ИЗВЕСТНЫМИ ИНВАРИАНТАМИ НА СУБКАНОНИЧЕСКОМ ПРЕОБРАЗОВАНИИ
}

В работе получено обобщенно-каноническое (субканоническое) преобразование, переводящее исходную неавтономную систему в две подсистемы, первая из которых принимает канонический вид, если импульсы второй - инварианты. В теоремах 1 и 2 даются достаточные условия для получения $2(n-r)$-мерной канонической подсистемы при наличии $r$ заданных или неизвестных инвариантов системы. Получено обращение принципа Мопертюи для систем с известным инвариантом и определены условия, при которых систему Гамильтона можно трактовать как систему Мопертюи. Результаты иллюстрируются на примере понижения порядка неавтономной системы Гамильтона с инвариантом-связкой гамильтониана и импульсов.

1. Рассмотрим неавтономную динамическую систему

$$
\begin{aligned}
& \dot{q}^{\prime \prime}=\partial G^{\prime \prime} / \partial p^{\prime \prime}, \quad \dot{p}^{\prime \prime}=-\partial G^{\prime \prime} / \partial q^{\prime \prime} \quad(\dot{f}=d f / d t), \\
& q^{\prime \prime}=\left(q_{\mu}\right)^{*}, \quad p^{\prime \prime}=\partial L^{\prime \prime} / \partial \dot{q}^{\prime \prime}=\left(p_{v}\right)^{*} \quad(\mu, v=1, n-2), \\
& L^{\prime \prime}=L^{\prime \prime}\left(t, q^{\prime \prime}, \dot{q}^{\prime \prime}\right), \quad\left|\partial^{2} L^{\prime \prime}\right| \partial \dot{q}_{\mu} \partial \dot{q}_{v} \mid \neq 0, \\
& G^{\prime \prime}=G^{\prime \prime}\left(t, q^{\prime \prime}, p^{\prime \prime}\right)=p^{\prime \prime} \cdot \dot{q}^{\prime \prime}-L^{\prime \prime}\left(t, q^{\prime \prime}, \dot{q}^{\prime \prime}\right),
\end{aligned}
$$

которой соответствует форма Пфаффа

$$
\omega_{d}^{\prime \prime}=p^{\prime \prime} \cdot d q^{\prime \prime}-G^{\prime \prime} d t .
$$

Введем $\left[{ }^{1-3}\right]$ новый параметр $\left(\tau^{\prime}\right)$

$$
d \tau^{\prime} / d t=v_{1}^{-1}\left(t, q^{\prime \prime}, p^{\prime \prime}\right), \quad 0<v_{1} \in C_{2},
$$

и новую функцию Гамильтона $G^{\prime}=v_{1}\left(p_{n-1}+G^{\prime \prime}\right)$ расширенной системы

$$
\begin{gathered}
d q^{\prime} / d \tau^{\prime}=\partial G^{\prime} / \partial p^{\prime}, \quad d p^{\prime} / d \tau^{\prime}=-\partial G^{\prime} / \partial q^{\prime}, \\
q^{\prime}=\left(q_{\mu}, t\right)^{*}, \quad p^{\prime}=\left(p_{v}, p_{n-1}\right)^{*}, \quad p_{n-1}=\partial v_{1} L^{\prime \prime} / \partial v_{1}=-G^{\prime \prime} .
\end{gathered}
$$

Уравнения (1.4) имеют частный интеграл $G^{\prime \prime}+p_{n-1}=0$, задающий в пространстве $q^{\prime}, p^{\prime}$ инвариантное множество $\Omega^{\prime \prime}$ системы (1.4), на котором (1.4) эквивалентна $(1.1),(1.3)$ и соотношению $G^{\prime \prime}=\partial G^{\prime \prime} / \partial t$. Система (1.1) погружена в $q^{\prime \prime}, t$-симметричную каноническую систему (1.4) с пфаффианом $\omega_{d}^{\prime}=p^{\prime} \cdot d q^{\prime}-G^{\prime} d \tau^{\prime}$. Для сохранения автономности системы (1.4) при $\partial G^{\prime \prime} / \partial t=0$ достаточно считать, что произвольная функция $v_{1,2}=v_{1,2}\left(q^{\prime \prime}, p^{\prime \prime}\right)$. В случае преобразования параметра $\tau^{\prime}$ операцию погружения следует повторить, вводя непреобразуемый параметр $\tau$ и функцию $H=G$ равенствами 


$$
d \tau / d \tau^{\prime}=v_{2}^{-1}\left(q^{\prime}, p^{\prime}\right), \quad p_{n}=\frac{\partial\left(v_{2} L^{\prime}\right)}{\partial v_{2}}=-G^{\prime}, \quad G=v_{2}\left(G^{\prime}+p_{n}\right) .
$$

Тогда получим 2n-мерную расширенную систему

$$
\begin{gathered}
d q / d \tau=\partial G / \partial p, \quad d p / d \tau=-\partial G / \partial q, \\
q=\left(q_{\mu}, t, \tau^{\prime}\right)^{*}, \quad p=\left(p_{v}, p_{n-1}, p_{n}\right)^{*}, \quad G=G\left(q^{\prime}, p\right),
\end{gathered}
$$

союзную с дифференциальной формой

$$
\omega_{d}(p, q)=p \cdot d q-G d \tau .
$$

Интегральные кривые системы (1.1) лежат в пространстве $(q, p)$ на пересечении $\Omega^{\prime \prime} \cap \Omega^{\prime}$ инвариантных множеств системы (1.5), соответствующих частным интегралам $h_{r-1}=p_{n-1}+G^{\prime \prime}=h_{r}=p_{n}=0$. Используя это обстоятельство, можно $\left[{ }^{1,2}\right]$, не уменьшая общности, вместо системы (1.1) с известными независимыми общими интегралами $h_{1}, h_{2}, \ldots, h_{r-2}$ и формой (1.2) рассматривать систему (1.5) с инвариантами $h_{\alpha}(q, p)(\alpha=\overline{1, r)}$ и формой (1.6). Далее для упрощения инварианты $h_{r-1}, h_{r}$ будем использовать как общие.

Предполагается, что $h_{\alpha}(x, p)$ - дважды непрерывно дифференцируемые функции, $\quad$ rang $\left\|\partial h_{\alpha} / \partial p_{k}\right\|=r \leqslant n, k=\overline{1, n}$.

2. Субканоническое преобразование переменных $q, p$ в $z=$ $=\left(x_{i}, \xi_{j}, y_{i}, \eta_{j}\right)$ * определим равенством дифференциальных форм

$$
\begin{gathered}
\omega_{d}(z) \equiv w y_{i} d x_{i}-H d \tau-v_{j} d \eta_{j}+d V=c\left(p_{k} d q_{k}-G d \tau\right), \\
x=\left(x_{i}\right)^{*}, \quad y=\left(y_{i}\right)^{*}, \quad \xi=\left(\xi_{j}\right)^{*}, \quad \eta=\left(\eta_{j}\right)^{*} \quad(i=1, n-r, j=1, r), \\
w=w(\eta), \quad v_{j}=v_{j}(\xi, \eta), \quad\left|\partial v_{j} / \partial \xi \alpha\right| \neq 0, \quad 0<w \in C_{2}, \quad v_{j} \in C_{2}, \\
H=H(\tau, x, \xi, y, \eta), \quad V=V(\tau, q, x, \eta), \quad c=\text { const, } \quad V \in C_{2},
\end{gathered}
$$

в котором функции $w, v_{j}$ произвольны, а $V$ удовлетворяет условию

$$
N_{0}=\left|\partial^{2} V / \partial q_{k} z_{l}^{\prime}\right| \neq 0, \quad z_{i}^{\prime}=x_{i}, \quad z_{n-r+j}^{\prime}=\eta_{j}, \quad l=\overline{1, n},
$$

обратимости отображения

$$
p=c^{-1} \frac{\partial V}{\partial q}, \quad y=-w^{-1} \frac{\partial V}{\partial x}, \quad v(\xi, \eta)=\frac{\partial V}{\partial \eta},
$$

заданного соотношением (2.1). Значение $H$ дает равенство

$$
H=c G+\frac{\partial V}{\partial t} \text {. }
$$

Субканоническое преобразование (2.3) принимает вид контактного преобразования С. Ли при $c=1, w \equiv 1, v \equiv \xi$ и отличается от канонического пресбразования $\left[{ }^{4}\right]$ условиями $w \neq$ const, $v \not \equiv \xi$. В силу равенства билинейных ковариантов

$$
\delta \omega_{d}(z)-d \omega_{\delta}(z)=c\left(\delta \omega_{d}(p, q)-d \omega_{\delta}(p, q)\right)=0
$$

получаем союзную для формы (2.1) систему

$$
\begin{gathered}
\frac{d x_{i}}{d \tau}=w^{-1} \frac{\partial H}{\partial y_{i}}, \quad \frac{\partial y_{i}}{d \tau}=-w^{-1}\left(\frac{\partial H}{\partial x_{i}}+\dot{w} y_{i}\right), \\
\frac{d \xi}{d \tau}=D^{*}\left[\frac{\partial H}{\partial \eta}-w^{-1}\left(y \cdot \frac{\partial H}{\partial y}\right) \frac{\partial w}{\partial \eta}\right]-A \frac{\partial H}{\partial \xi}, \quad \frac{d \eta}{d \tau}=-D \frac{\partial H}{\partial \xi},
\end{gathered}
$$




$$
\begin{gathered}
B=\left\|b_{\alpha j}\right\|, \quad b_{\alpha j}=\frac{\partial v_{j}}{d \xi_{\alpha}}, \quad D=B^{-1}, \quad \dot{w}=\frac{d w}{d \tau}, \\
A=D^{*} C D, \quad A^{*}=-A, \quad C=\left\|C_{\alpha j}\right\|, \quad C_{\alpha j}=\frac{\partial v_{\alpha}}{\partial \eta_{j}}-\frac{\partial v_{j}}{\partial \eta_{\alpha}},
\end{gathered}
$$

в которую преобразование (2.3) переводит уравнения (1.5).

Из структуры подсистемы (2.6) новых уравнений выводится

Ле м м а. Функция $H$ субканонической системы (2.5), (2.6) не зависит от вектора э тогда и только тогда, когда величины $\eta_{j}=g_{j}(\tau, q, p)$, данные преобразованием (2.3), являются независимыми общими инвариантами системы (1.5).

Д ок а зательство. Если $g_{j}(\tau, q, p)$ - инварианты системы $(1.5)$, то $\eta_{j}$ - инварианты системы (2.5), (2.6). В силу $|D| \neq 0$ последнее возможно лишь в случае $\partial H / \partial \xi=0$. Необходимость установлена. Если имеем $\partial H / \partial \xi=0$, то $g_{j}(\tau, q, p)$ - инварианты системы (1.5), независимые по условиям $(2.1),(2.2)$. Лемма доказана.

Следстви е 1: подсистема типа Стрэтта (2.5) приводится к каноническому виду

$$
\begin{aligned}
& d x / d \sigma=\partial H / \partial y, \quad d y / d \sigma=-\partial H / \partial x, \\
& d \sigma / d \tau=w^{-1}, \quad H=H(\sigma, x, y \mid \eta), \quad \eta=\mathrm{const},
\end{aligned}
$$

если величины $\eta$, заданные субканоническим преобразованием (2.1), являются независимыми инвариантами системы (1.5).

Поставим задачу - установить условия, при которых субканоническое преобразование $(2.1)$ даст $2(n-r)$-мерную каноническую подсистему (2.5) для любых фиксированных значений известных инвариантов $h^{0} \alpha=h_{\alpha}\left(q^{0}, p^{0}\right), q^{0}=q\left(\tau^{0}\right), p^{0}=p\left(\tau^{0}\right)$.

Для решения задачи воспользуемся инвариантами $h_{\alpha}$. Предположим, что существует субканоническое преобразование $(2.1)-(2.3)$, для которого одновременно выполняются равенства

$$
\eta_{j}=h_{j}(q, p), \quad \text { rang }\left\|\partial h_{\alpha} / \partial p_{k}\right\|=r, \quad j=\overline{1, r} .
$$

Допущение означает, что отображение (2.3) переводит известные инварианты $h$ системы (1.5) в импульсы $\eta$ новой системы (2.5), (2.6).

Ввиду соотношений (2.3) условие (2.8) равносильно уравнениям

$$
f_{j}(q, \psi) \equiv h_{j}\left(q, c^{-1} \psi\right)=\eta_{j} \quad(\psi=\partial V / \partial q),
$$

в которых $\eta_{j}$ рассматриваются как произвольные и независимые параметры $\eta_{j}=\eta_{j}^{0}=h_{j}\left(q^{0}, p^{0}\right)$. Система (2.9) должна быть совместной по предположению. В силу независимости $\eta_{j}$ и свойств $h_{\alpha}(q, p)$ необходимо $\left[{ }^{5,6}\right]$, чтобы все коммутаторы $f_{\alpha j}$ системы (2.9) тождественно исчезали для $V=V(\tau, q, x, \eta)$. Отсюда выводим необходимые для совместности системы (2.9) уравнения

$$
\begin{gathered}
f_{\alpha j}(q, \psi)=\left[f_{\alpha}, f_{j}\right]=\frac{\partial f_{\alpha}}{\partial q_{k}} \frac{\partial f_{j}}{\partial \psi_{k}}-\frac{\partial f_{j}}{\partial q_{k}} \frac{\partial f_{\alpha}}{\partial \psi_{k}}=\frac{1}{c}\left(h_{\alpha}, h_{j}\right)=0, \\
\left(h_{\alpha}, h_{j}\right)=\left(\frac{\partial h_{\alpha}}{\partial q_{k}} \frac{\partial h_{j}}{\partial p_{k}}-\frac{\partial h_{j}}{\partial q_{k}} \frac{\partial h_{\alpha}}{\partial p_{k}}{ }_{p=c^{-1} \psi} .\right.
\end{gathered}
$$

Использовав коммутаторы для независимой подсистемы в системе 
уравнений $(2.9),(2.10)$ и всех последующих, построим полную якобиеву систему $\left[{ }^{5-7}\right]$ независимых уравнений $F$

$$
f_{j}=\eta_{j}, \quad f_{m}^{\prime}=0 \quad(j=\overline{1, r}, \quad m=\overrightarrow{r+1, s)}
$$

ранга $s$ для совместной системы $(2.9)$, где $r \leqslant s \leqslant n$. Тогда на основании следствия 1 получим следующий результат.

Те орем а 1. Eсли инварианты (2.8) u постоянная $c=c_{*} \neq 0$ определяют полную совместную систему (2.11), которая имеет решение $V=V *(\tau, q, x, \eta)$, удовлетворяющее условиям (2.1), (2.2), то существует субканоническое преобразование (2.3), приводящее подсистему (2.5) $\kappa$ каноническому виду (2.7) с рангом $2(n-r)$.

Полученное утверждение является модификацией теоремы С. Ли [ $\left.{ }^{7}\right]$ о возможности понижения порядка канонической системы на $2 r$ единиц при наличии $r$ инвариантов $(2.8)$, которые находятся в инволюции $\left(h_{\alpha}, h_{j}\right) \equiv 0$. В этом случае система уравнений (2.11) совпадает с (2.9) и $c \equiv 1$. Теорема 1 распространяет результат С. Ли на субканоническое

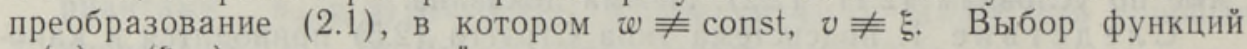
$w(\eta), v(\xi, \eta)$ и постоянной $c$ можно использовать для упрощения вида $2 r$-мерной подсистемы (2.6) и применения методов линеаризации, разделения переменных и малого параметра в целях ее интеграции.

Таким образом, вид (2.3) искомого преобразования задачи дает решение $V$ * системы (2.11) со свойствами (2.1), (2.2). При этом интеграция подсистемы (2.5), сводимой к (2.7), проводится при $\eta=\eta^{0}$ независимо от подсистемы (2.6).

Поставим другую задачу - найти при неизвестных инвариантах системы (1.5) субканоническое преобразование, для которого интеграцию подсистемы (2.5) можно проводить отдельно от подсистемы (2.6).

Следствие 2: подсистема (2.5) не связана с $(2.6)$, если величина (2.4) не зависит от $\xi$.

Действительно, из леммы для подсистем (2.5), (2.6) заключаем, что в этом случае (2.5) сводится к виду (2.7) преобразованием параметра $d \tau=w d \sigma$ и поэтому ее интеграция не связана с подсистемой (2.6).

Отсюда следует, что для решения этой задачи достаточно определить условия, которые дадут равенства $\partial H / \partial \xi=0$.

Легко убедиться в инвариантности искомых условий относительно допустимого выбора вида $v_{j}(\xi, \eta)$ при фиксированных функциях $V$, w. Это означает, что $\partial H / \partial \xi=0$ лишь в случае $\partial H^{*} / \partial v=0$, где $H^{*}(\tau, x, v(\xi, \eta), y, \eta) \equiv H(\tau, x, \xi, y, \eta)$. Из уравнений (2.6) для циклических $\xi_{j}^{*}$ (при выборе $\left.v \equiv \xi\right)$ имеем, как и в общем случае, $\eta_{j}=$ const. Поэтому, не уменьшая общности, положим здесь $v_{j}(\xi, \eta) \equiv \xi$. Введем обозначения

$$
N=\left\|n_{l k}\right\|, \quad n_{l k}=\frac{\partial^{2} V}{\partial z_{l}^{\prime} \partial q_{k}}, \quad R=\left\|r_{k j}\right\|, \quad r_{h j}=\frac{\partial q_{k}}{\partial \xi_{j}} .
$$

Продифференцировав по $\xi_{j}$ соотношения (2.3), получим матричное равенство $R=N^{-1} I$, где

$$
N^{-1}=\left\|n_{l k}^{\prime}\right\|, \quad n_{l k}^{\prime}=N_{0}^{-1} P\left(n_{k l}\right), \quad I=\left\|\begin{array}{c}
0 \\
E_{r}^{r}
\end{array}\right\|,
$$

$P\left(n_{k l}\right)$ - алгебраическое дополнение элемента $n_{k l}$ в $N, \quad N_{0}=\operatorname{det} N$, $r_{k j}=R_{k j}\left(n_{l k}\right)$ - рациональные функции. Условия $\partial H / \partial \xi=0$ эквивалентны системе $r$ уравнений 


$$
\left[\frac{\partial^{2} V}{\partial \tau \partial q_{l}}+c\left(\frac{\partial G}{\partial q_{l}}\right)+\left(\frac{\partial G}{\partial p_{k}}\right) \frac{\partial^{2} V}{\partial q_{k} \partial q_{l}}\right] r_{l j}=0
$$

которым должна удовлетворять функция $V$. Тогда из следствия 2 и системы (2.12), где символ $\left(\partial G / \partial r_{l}\right)$ означает функции после подстановки $\xi=\partial V / \partial \eta, y=-w^{-1} \partial V / \partial x$, имеем утверждение, которое выражает

Те орема 2. Если при $c=c^{*}$ существует решение $V^{*}$ системь (2.12), удовлетворяющее условиям (2.1), (2.2), то оно определяет субканоническое преобразование (2.3), которое дает подсистему (2.5), интегрируемую отдельно от подсистемь (2.6).

При этом функции $g_{j}(\tau, p, q)=\eta_{j}$ являются независимыми инвариантами, вид которых дает преобразование (2.3).

3 а м е ч а н я. Используемое здесь погружение системы (1.1) в систему (1.4) для учета случая субканонического преобразования времени в симметричной форме не является необходимостью и вполне заменимо соответствующей модификацией канонического преобразования вида $\left[{ }^{8}\right]$. Аналогично методу Якоби, эффективность условий двойного понижения порядка в теоремах 1 и 2 зависит от структуры инвариантов $h_{\alpha}$ и функции $G$. Для циклических координат $q^{\prime \prime}, t$ исходной системы (1.1) такое понижение дается известными уравнениями Раута и Уиттекера. В последнем случае эволюция динамической системы со структурным свойством $\partial G^{\prime \prime} / \partial t=0$ и инвариантом $G^{\prime \prime}\left(q^{\prime \prime}, p^{\prime \prime}\right)$ определяется принципом Мопертюи. В связи с этим для неавтономных динамических систем интересно привести следующую модификацию принципа Мопертюи.

3 . Рассмотрим $2 n$-мерную с инвариантом $h(t, x, \dot{x})$ динамическую систему $S$, эволюция которой выражается условиями стационарности функционала $I=\int_{t_{0}}^{t_{*}^{*}} M(t, x, \dot{x}) d t \quad$ при ограничении $h[t, x(t), \dot{x}(t)]=$ $=h\left(t_{0}, x\left(t_{0}\right), \quad \dot{x}\left(t_{0}\right)\right)=h_{0}$ и краевых условиях $x\left(t_{0}\right)=x_{0}, x\left(t_{*}\right)=x_{1}$. Предполагается, что $t_{0}, x_{0}, x_{1}$ - произвольны и фиксированы, $t$ * - не задано, определитель

$$
D_{1}=\left|\partial^{2}(M+\lambda h) / \partial \dot{x}_{k} \partial \dot{x}_{s}\right| \neq 0 \quad \text { и } \quad \dot{x}_{s} \frac{\partial h}{\partial \dot{x}_{s}} \neq 0 \quad(k, s=\overline{1, n}) .
$$

Условия стационарности $I$ имеют вид

$$
\begin{gathered}
\frac{d}{d t}\left[\frac{\partial}{\partial \dot{x}}(M+\lambda h)\right]-\frac{\partial}{\partial x}(M+\lambda h)=0, \\
\frac{\partial h}{\partial t}+\dot{x} \cdot \frac{\partial h}{\partial x}+\ddot{x} \cdot \frac{\partial h}{\partial \dot{x}}=0, \\
\lambda_{*}\left[H \cdot(h)+h_{0}\right]+H \cdot(M)=0, \\
H(\Phi) \equiv \dot{x} \cdot \frac{\partial \Phi}{\partial \dot{x}}-\Phi, \quad \Phi *=\Phi\left(t_{*}, x\left(t_{*}\right), \dot{x}\left(t_{*}\right)\right), \lambda=\lambda(t) .
\end{gathered}
$$

Допустим также, что данная неавтономная система удовлетворяет структурным условиям, которые заменяют для $M, h$ условия цикличности

$$
\frac{\partial h}{\partial t} H(M)-[h+H(h)] \frac{\partial M}{\partial t}=0, \quad c_{1}=\mathrm{const} \neq h^{-1},
$$




$$
\left(1-c_{1} h\right)[h+H(h)]^{-1} H(M)+c_{1} M=\dot{V}_{1}(t, x), \quad V_{1} \in C_{2} .
$$

Из первых $n+1$ уравнений системы (3.1) получаем, что множитель Лагранжа $\lambda(t)$ удовлетворяет уравнению

$0=\dot{\lambda}+[H(h)+h]^{-1}\left\{\left[\frac{d}{d t}(H(h)+h)+\frac{\partial h}{\partial t}\right] \lambda+\frac{d}{d t} H(M)+\frac{\partial M}{\partial t}\right\}$,

которое в силу первого равенства (3.2) имеет решение

$$
\lambda=\mu(t, x, \dot{x})=-[h+H(h)]^{-1} H(M),
$$

удовлетворяющее краевому условию в системе (3.1).

Определим для $S$ функцию $L^{*}(t, x, \dot{x})$ равенством

$$
c_{2} L^{*}=M+\mu h+\hat{V}_{2}, \quad V_{2}=V_{2}(t, x) \in C_{2}, \quad c_{2}=\text { const } \neq 0,
$$

где $V_{1,2}(t, x)$ - произвольная функция. Отсюда имеем следующее утверждение.

Если $D_{2}=\left|\partial^{2} L^{*} / \partial \dot{x}_{k} \partial \dot{x}_{s}\right| \neq 0$, то система $S$ - гамильтонова. В справедливости этого утверждения убеждаемся подстановкой выражения (3.5) в уравнение (3.1), которая приводит с учетом (3.2) к уравнениям خйлера-Лагранжа, определяющим эволюцию системы $S$ при $D_{2} \neq 0$. Функция $h\left(t, x, \partial H^{*} / \partial p\right)$ является инвариантом $h^{\prime}(t, x, p)$ системы с гамильтонианом $H^{*}=H^{*}(t, x, p)=H\left(L^{*}\right)$. Этот вывод можно рассматривать как обращение принципа Мопертюи.

Обратно, пусть для данной функции $H^{*}$ системы Гамильтона с инвариантом $h^{\prime}(t, x, p)$ существует достаточно гладкое решение $M(t, x, \dot{x})$ уравнений (3.2), (3.5) при $\mu$, заданном равенством (3.4). Если $D_{1} \neq 0$, то $M(t, x, \dot{x})$ будет подынтегральной функцией обобщенного принципа Мопертюи.

4. Прим е ры. Рассмотрим неавтономную гамильтонову систему

$$
\begin{aligned}
& \dot{q}=\partial G / \partial p, \quad \dot{p}=-\partial G / \partial q, \quad q=\left(q_{s}\right)^{*}, \quad p=\left(p_{k}\right)^{*}, \\
& G=1 / 2 g_{s k}(t, q) p_{s} p_{k}+g_{s}(t, q) p_{s}+G_{0}(t, q), \quad\left\|g_{s k}\right\|>0
\end{aligned}
$$

структура которой удовлетворяет условию [9 $]$

$$
\frac{\partial G}{\partial t}-u_{k} \frac{\partial G}{\partial q_{k}}+\dot{u}_{k} p_{k}-v=0
$$

содержащему заданные функции $u_{k}(t), v(t)$ класса $C_{2}$.

В силу равенства (4.2) система (4.1) имеет инвариант

$$
h^{\prime}=F^{\prime}=G+u_{k}(t) p_{k}-W(k, s=\overrightarrow{1, n}),
$$

где $\quad g_{s k}(t, q) \equiv g_{s k}(x), \quad g_{s}(t, q) \equiv f_{s}(x)-u_{s}(t), \quad W=\int_{0}^{t} v d t$,

$-W(t)+G_{0}(t, q) \equiv G_{0}(x), \quad x=q+w, \quad w=\int_{0}^{t} u d t, \quad f_{s}-$ некоторые функции класса $C_{2}$.

Функция $V(t, q, P)=x_{k} P_{k}-\int_{0}^{t} W(\tau) d \tau$ определяет новые переменные

$$
\begin{aligned}
Q_{k} & =\partial V / \partial P_{k}=q_{k}+w_{k}=x_{k}, \\
p_{k} & =\partial V / \partial q_{k}=P_{k},
\end{aligned}
$$




$$
H=G+\partial V / \partial t=G+-u_{k} p_{k}-W(t)=F^{\prime},
$$

в которых система (4.1) с инвариантом (4.3) примет вид канонических уравнений

$$
\begin{aligned}
& \dot{x}=\partial F^{\prime} / \partial p, \quad \dot{p}=-\partial F^{\prime} / \partial x, \\
& \left(\dot{F}^{\prime}=\partial F^{\prime} / \partial t=0\right) .
\end{aligned}
$$

Обозначим решение уравнения $F^{\prime}(x, p)+h^{0}=0$ относительно $p_{1}$ через $-K\left(x, p_{2}, \ldots, p_{r}, h^{0}\right)$. Отсюда имеем систему Уиттекера

$$
\begin{array}{ll}
\frac{d x_{i}}{d x_{1}}=\frac{\partial K}{\partial p_{i}}, & \frac{d p_{i}}{d x_{1}}=-\frac{\partial K}{\partial x_{i}}, \\
\frac{d t}{d x_{1}}=\frac{\partial K}{\partial h^{0}}, \quad h^{0}=\text { const }, \quad i=2,3, \ldots, n,
\end{array}
$$

в которой первые $2(n-1)$ уравнений имеют вид $(2.7)$ и интегрируются независимо от двух последних. Неавтономная система (4.1) со свойством (4.2) служит примером реализации условий понижения размерности системы, полученных в п. 2. В работе $\left[{ }^{9}\right]$ приводятся примеры механических систем (гиростатов), для которых условие (4.2) выполнено. С помощью инварианта (4.3) размерность этих систем, согласно примеру, можно уменьшить на две единицы. Существенно отметить, что при этом система (4.1) не обязана быть натуральной.

Примером выполнения условий п. 3 является система МопертюиЛагранжа

$$
\begin{aligned}
& M=2 T=a_{k s}(x) \dot{x}_{k} \dot{x}_{s}, \quad L=T+U, \\
& U=U(x), \quad x=\left(x_{k}\right)^{*}, \quad k, s=\overline{1, n} .
\end{aligned}
$$

Так как инвариант движения $h=T-U$ и функция $M$ не зависят от $t$, то условие (3.2) выполнено. Для этой системы по формуле (3.4) находим $\mu=-1$. Тогда равенство (3.5) удовлетворяется. Поэтому сба утверждения п. 3 справедливы в этом примере.

\section{ЛИТЕРА Т У А}

1. С ин г Дж. Л., Классическая механика, М., 1963.

2. Бергман П. Г., Введение в теорию относительности, М., 1947.

3. П а р с Л. А., Аналитическая динамика, М., 1971.

4. Г а н т ахер Ф. Р., Лекции по аналитической механике, М., 1966.

5. Э й зенх а рт Л. П., Непрерывные группы преобразований, М., 1947.

6. В а лле-Пуссен Ш. Ж., Лекции по теоретической механике, т. II, М., 1949.

7. Леви-Чивита Т., Амальди У., Курс теоретической механики, т. II, ч. 2, M., 1951.

8. Н о воселов В. С., Механика твердого тела, № 5,16 (1974).

9. Кей с И., Изв. АН ЭССР, Физ. Матем., 24, 277 (1975).

Ннститут кибернетики

Академии наук Эстонской ССР
Поступила в редакцию $24 / \mathrm{VI} \quad 1975$ 


\section{KEIS}

\section{SUBKANOONILISEL TEISENDUSEL INVARIANTE OMAVATE HAMILTONI SUSTEEMIDE JÄRGU ALANDAMISEST}

Vaadeldakse üldistatud kanoonilist (subkanoonilist) teisendust, mis muudab esialgse dünaamilise süsteemi kaheks alamsüsteemiks. Esimesel süsteemil on kanooniline kuju, kui teise süsteemi impulsid on invariantsed. Teoreemidega 1 ja 2 on kindlaks määratud piisavad tingimused $2(n-r)$-mõõtmelise kanoonilise alamsüsteemi saamiseks nii etteantud kui ka tundmatute invariantide korral. On saadud Maupertuis' printsiibi pöördprintsiip mōnede tuntud invariantidega süsteemide jaoks. Määratakse tingimused, mille korral Hamiltoni süsteemi võib esitada Maupertuis' süsteemina. Tuuakse näide mõningate mitteautonoomsete Hamiltoni süsteemide (hamiltoniaani ja impulsside lineaarsete kombinatsioonide) järgu alandamise kohta.

\section{KEIS}

\section{DIMENSION REDUCTION VIA SUBCANONICAL TRANSFORM OF HAMILTONIAN SYSTEMS WITH INVARIANTS}

Subcanonical transform reducing initial system to special combination of two subsystems is introduced in the paper. The first one becomes canonical when the impulses of the second system are invariants, Existence conditions of subcanonical transform resulting in a $2 r$-dimension reduction of the system are proved in theorems 1,2 . Maupertuis' inverse principle for the system with invariant is put forward. Conditions when the Hamiltonian system can be treated as Maupertuis' system are also determined. 